\title{
TECHNOLOGY ROADMAPPING COM APLICAÇÃO PRESENCIAL E TELEPRESENCIAL - UM ESTUDO COMPARATIVO
}

Carla Santos de Souza Giordano (SSOUZA@firjan.com.br) - Federação das Indústrias do Rio de Janeiro (Firjan).

Joana da Fonseca Rosa Ribeiro (joaribeiro@firjan.com.br) - Federação das Indústrias do Rio de Janeiro (Firjan).

Andressa Oliveira Costa de Jesus (aojesus@firjan.com.br) - Federação das Indústrias do Rio de Janeiro (Firjan).

\section{RESUMO}

Desde 2008, a Firjan SENAI aplica o método Technology Roadmapping como ferramentas de gestão para estruturar e sistematizar o planejamento tecnológico dos seus Institutos. Desde então, a Rota Tecnológica passou por diversos momentos de adequação diante do contexto de constantes mudanças observadas nos últimos anos a fim de garantir aproveitamento satisfatório no processo de construção do roadmap. O objetivo do presente estudo é avaliar as transformações ocorridas no processo de construção das Rotas Tecnológicas dos Institutos SENAI de Inovação e Tecnologia Química e Meio Ambiente nos últimos 10 anos e consequente identificação das inovações e aprendizados advindos deste contexto, em destaque para o último ciclo de atualização realizado durante o cenário de distanciamento social que contribuiu para massificação do trabalho em modelo telepresencial vivido em 2020. A metodologia aplicada no presente artigo consiste em um processo comparativo entre a construção do roadmap 2011-2015, realizado em 2010 e o roadmap 2021-2025 realizado em 2020. Os resultados indicam boas expectativas com a realização de um modelo híbrido, que considera ações presenciais e telepresenciais como tendência para os próximos anos, resguardado as adaptações necessárias.

Palavras chave: Technology Roadmap; Abordagem Digital; Estudo Comparativo, presença versus telepresença, Modelo Híbrido. 


\section{INTRODUÇÃ̃o}

Nos últimos anos o desenvolvimento tecnológico ocorreu de forma acelerada e passamos de um cenário onde 'todos podem' ficar online para um cenário onde 'todos estão' online a todo tempo e em todo lugar (WEFORUM, 2021). Posto isto, e em evidente destaque, os últimos 10 anos marcaram-se por mudanças disruptivas que provocaram consequências na forma como nos relacionamos com a tecnologia, no qual a interação antes realizada por meio de dispositivos móveis passou a utilizar realidade virtual; Informações e dados antes obtidos por análises preditivas agora são extraídos por meio de automação cognitiva; E a computação por si, passou de arquitetura em nuvens para distribuição em diversas plataformas.

Este cenário, impulsionado e expandido pelos efeitos provocados pela pandemia do Covid-19, trouxe desafios para as empresas e a sociedade, em destaque para a forma como se planejar e se preparar para o futuro diante da transformação na relação homem-máquina em busca do formato ideal: hora presencial, hora telepresencial; Onde a telepresença surge como uma possibilidade efetiva de se fazer presente (independente da distância geográfica), pois este permite a ação e a interação dos sujeitos nesse mundo conectado, fazendo surgir um tipo de vida denominado por muitos teóricos de "vida digital virtual" (SCHLEMMER, 2009).

Por outro lado, esta interação com os dispositivos é construída de forma generalizada, cruzando diferentes dispositivos em experiências cross-channel (BENYON E RESMINI 2017), ou seja, que podem viabilizar processos híbridos que mesclam espaços digitais e físicos (RENZI et al., 2020). Nesse sentido, adaptar o processo de mapeamento e planejamento tecnológico a este ambiente é fator decisivo para viabilizar qualquer plano estratégico.

Assim, o acompanhamento das mudanças aplicadas à metodologia de roadmapping tecnológico e a inclusão de uma abordagem digital no processo, vem a ser um tema de grande relevância para empresas que buscam fazer uso do método em seus respectivos planejamentos. Ademais, diante do recente engrandecimento do ambiente de trabalho telepresencial nas atividades empresariais e acadêmicas, a aplicação de uma metodologia de roadmapping com aplicação híbrida (presencial e telepresencial) em uma empresa consiste em um tema inovador. 


\subsection{O Objeto de Estudo}

O objeto deste estudo é a metodologia Technology Roadmapping, adaptada para estruturar e sistematizar o planejamento estratégico tecnológico do Instituto SENAI de Inovação (ISI) Química Verde e do Instituto SENAI de Tecnologia (IST) em Química e Meio Ambiente da Federação das Indústrias do Rio de Janeiro - Firjan, localizada no Estado do Rio de Janeiro Brasil, onde o ISI tem o objetivo de promover o crescimento sustentável da indústria brasileira, em médio e longo prazo por meio de pesquisa, desenvolvimento e inovação (PD\&I), e o IST auxilia a indústria na melhoria da condição ambiental por intermédio da prestação de consultoria, serviços de metrologia, análises e infraestrutura laboratorial.

\subsection{O Objetivo do Estudo}

O objetivo deste estudo é avaliar as transformações ocorridas no Technology Roadmap construído no ano de $2020^{1}$, ano marcado pelo contexto pandêmico e pelas significativas alterações na forma de trabalho, exercendo uma contraposição comparativa da metodologia realizada em $2010^{2}$ e destacar as principais inovações e aprendizados advindos desta transformação.

\section{METODOLOGIA}

\subsection{O Technology Roadmapping}

O roadmap é uma abordagem que busca capturar uma visão sintetizada e integrada do plano estratégico em um formato simples, que relaciona mercado, produto e tecnologia (PHAAL, FARRUKH e PROBERT, 2005). Já o Technology Roadmapping - TRM é o método que pode ser utilizado para diferentes propósitos e abrangências, passando por empresas específicas, setores industriais e mesmo, políticas governamentais de desenvolvimento e tecnologia.

\footnotetext{
${ }^{1}$ para o horizonte temporal 2021-2025.

2 para o horizonte temporal 2011-2015.
} 
Este método foi aplicado e reconhecido por instituições de grande influência nos últimos anos, sendo considerado pelas nações unidas uma ferramenta capaz de definir direções e orientar ações associadas aos objetivos das instituições (UNIDO, 2005; KERR E PHAAL, 2020). Por outro lado, a vasta aplicabilidade do método TRM repercute em desafios consideráveis para as empresas que necessitam adaptá-la de acordo com sua atuação e objetivos pois embora a figura final, o roadmap, se apresente de forma simples em estrutura e conceito, a coleta e priorização das informações nele contidas são fruto de um processo de estratégia e planejamento que é ponto forte do método (PHAAL, FARRUKH AND PROBERT, 2001; GARCIA E BRAY, 1997).

Desta forma, o Technology Roadmapping é utilizado pela Firjan SENAI como método de Planejamento Estratégico Tecnológico (GIORDANO, 2011). Aplicado desde $2008^{3}$ permite conhecer as necessidades atuais e futuras dos Institutos de Inovação e Tecnologia e sua continuidade e fluidez são a base para manter um portfólio de pesquisa, desenvolvimento e inovação (PD\&I) e serviços de tecnologia atualizado, que visa contribuir para um ambiente cada vez mais favorável à inovação.

\subsection{As Etapas do Technology Roadmapping e o Modelo de Atendimento}

A metodologia Technology Roadmapping adaptada para o objeto em estudo como método de Planejamento Estratégico Tecnológico, prevê sua construção ao longo de 6 etapas: Planejamento, Pesquisa, Mercado, Produto, Tecnologia e Mapeamento. Apenas as etapas de Mercado e Tecnologia contam com a realização de encontros com convidados externos à Instituição, onde a etapa de Mercado é constituída em 2 momentos, designados Mercado Externo (com especialistas não integrante do grupo profissional da Instituição) e Mercado Interno (com especialistas integrantes do grupo profissional da Instituição). As demais consistem em atividades somente com profissionais internos.

Apenas no ano de 2020, a partir da etapa de Tecnologia, a construção do roadmap ocorreu de forma exclusivamente telepresencial, no qual os workshops foram substituídos por webinars e reuniões online, representado na Tabela 1.

\footnotetext{
${ }^{3}$ A partir do ano de 2010 adotou-se o ciclo de 5 anos para se estruturar o Technology Roadmapping conforme período estabelecido para o Planejamento Estratégico da Instituição.
} 
TABELA 1 - Modelo de atendimento em cada etapa na construção do roadmap.

\begin{tabular}{l|l|l}
\hline Etapa & Roadmap 2011-2015 & Roadmap 2021-2025 \\
\hline Planejamento & presencial & presencial \\
\hline Pesquisa & presencial & presencial \\
\hline Mercado* & presencial & presencial \\
\hline Produto & presencial & presencial \\
\hline Tecnologia* & presencial & telepresencial \\
\hline Mapeamento & presencial & telepresencial \\
\hline
\end{tabular}

\section{RESULTADOS E DISCUSSÃO}

Perante contextualização, o caso em tela apresentou os seguintes resultados após estudo comparativo, aprofundadas a seguir:

- Modificação do perfil da equipe idealizadora.

- Novas ferramentas de trabalho direcionadas ao uso digital.

- Transformação decorrente do trabalho em telepresença.

- Variação do esforço de investimento.

- Renovação do modelo de entrega.

\subsection{Modificação do Perfil da Equipe Idealizadora}

Uma das primeiras alterações percebidas foi na composição da equipe idealizadora do estudo, designada equipe facilitadora. No ano de 2010 a equipe facilitadora contou com 1 profissional de nível estratégico além do líder do processo. Já no ano de 2020, a equipe foi composta por 1 profissional de nível operacional, 1 profissional de nível tático, 1 profissional com foco em design e 1 pesquisador bolsista de dedicação exclusiva ao projeto, além do próprio líder do processo.

No caso em tela, a presença do pesquisador bolsista foi possível devido ao programa de incorporação de pesquisadores bolsistas do Objeto em estudo que visa fomentar e apoiar a 
pesquisa direcionada a projetos de interesse institucional, cujo foco é desenvolver conhecimento técnico científico aplicado à realidade da indústria, em consonância com o interesse do bolsista. A Tabela 2 representa a alteração no perfil das equipes de ambos ciclos.

TABELA 2 -Perfil da equipe participante do processo

\begin{tabular}{|c|c|c|}
\hline Equipe & Roadmap 2011-2015 & Roadmap 2021-2025 \\
\hline Líder & $\begin{array}{l}\text { Notório saber e comprovada } \\
\text { experiência no processo } \\
\text { construtivo do roadmap }\end{array}$ & $\begin{array}{l}\text { Notório saber e comprovada } \\
\text { experiência no processo } \\
\text { construtivo do roadmap }\end{array}$ \\
\hline Facilitadora & $\begin{array}{l}1 \text { profissional de nível } \\
\text { estratégico. }\end{array}$ & $\begin{array}{l}\text { - } 1 \text { pesquisador bolsista de } \\
\text { dedicação exclusiva. } \\
\text { - } 1 \text { especialista de gestão } \\
\text { tecnológica de dedicação } \\
\text { compartilhada. } \\
\text { - } 1 \text { profissional técnico de nível } \\
\text { operacional. } \\
\text { - } 1 \text { profissional de design }\end{array}$ \\
\hline Técnica & $\begin{array}{l}\text { Profissionais da instituição, } \\
\text { especialistas e analistas } \\
\text { responsáveis por tarefas } \\
\text { operacionais. }\end{array}$ & $\begin{array}{l}\text { Profissionais da instituição, } \\
\text { especialistas e analistas } \\
\text { responsáveis por tarefas } \\
\text { operacionais. }\end{array}$ \\
\hline
\end{tabular}

Observa-se que o principal aprendizado advindo da adoção de uma equipe facilitadora com maior quantidade de profissionais foi:

- Ganhos em análise crítica dos processos diante da presença de uma equipe multidisciplinar.

\subsection{Novas Ferramentas de Trabalho Direcionadas ao Uso Digital}

Na sequência, observou-se significativa variação de uso e disponibilidade de ferramentas de trabalho entre ciclos. Na etapa de planejamento do ciclo 21-25, compreendeu-se que seria relevante utilizar ferramentas auxiliares capazes de apoiar a coleta de informações e tomada de decisões. Estas foram selecionadas durante o planejamento de cada etapa e de acordo com a necessidade e o formato definido para cada tarefa.

Nota-se que cada ferramenta possui diferentes objetivos como aumento do engajamento dos participantes durante a realização dos eventos, busca por diferentes formas de representações visuais, atendimento ao ambiente ágil, melhor tangibilização dos resultados para os 
stakeholders, e por fim, a viabilização da continuidade do processo no cenário de distanciamento social. Essas ferramentas estão representadas na Tabela 3 a seguir.

TABELA 3- Ferramentas utilizadas por Etapa

\begin{tabular}{|c|c|c|c|c|}
\hline \multirow{2}{*}{ Etapas } & \multicolumn{2}{|c|}{ Ciclo 2011-2015 } & \multicolumn{2}{|c|}{ Ciclo 2021-2025 } \\
\hline & Ferramentas & Resultado esperado & Ferramentas & Resultado esperado \\
\hline $\begin{array}{c}\text { Planejamento } \\
\text { e Pesquisa }\end{array}$ & Pacote Office & $\begin{array}{l}\text { Organização da } \\
\text { programação }\end{array}$ & Pacote Office & $\begin{array}{l}\text { Organização da } \\
\text { programação }\end{array}$ \\
\hline \multirow{7}{*}{$\begin{array}{l}\text { Mercado, } \\
\text { Produto e } \\
\text { Tecnologia }\end{array}$} & $\begin{array}{l}\text { Software } \\
\text { MindManager } 24\end{array}$ & $\begin{array}{l}\text { Suporte a discussão e } \\
\text { consenso nas dinâmicas }\end{array}$ & Coggle & $\begin{array}{l}\text { Organização do } \\
\text { pensamento e suporte para } \\
\text { consenso nas dinâmicas }\end{array}$ \\
\hline & $\begin{array}{l}\text { Flip charts e } \\
\text { Post-it® }\end{array}$ & $\begin{array}{l}\text { Organização do } \\
\text { pensamento }\end{array}$ & $\begin{array}{l}\text { Flip charts e } \\
\text { Post-it@ }\end{array}$ & $\begin{array}{l}\text { Organização do } \\
\text { pensamento }\end{array}$ \\
\hline & & & Microsoft ${ }^{\circledR}$ Teams & Encontros telepresencial \\
\hline & & & Storytelling & $\begin{array}{l}\text { Aumento do engajamento } \\
\text { dos participantes durante } \\
\text { a realização dos eventos }\end{array}$ \\
\hline & & & Pacote Office & Apresentação de conteúdo \\
\hline & & & Vídeos Mp4 & $\begin{array}{l}\text { Novas formas de } \\
\text { representação visual }\end{array}$ \\
\hline & & & Google forms & Obtenção de dados \\
\hline \multirow[b]{2}{*}{ Mapeamento } & Microsoft $®$ Visio & Representação gráfica & Pacote Adobe $\AA$ & Representação gráfica \\
\hline & Pacote Office & $\begin{array}{l}\text { Organização dos } \\
\text { resultados }\end{array}$ & Pacote Office & $\begin{array}{l}\text { Organização dos } \\
\text { resultados }\end{array}$ \\
\hline
\end{tabular}

A maior transformação observada foi obtida pela inserção do meio digital durante o mapeamento que ocorreu de forma decisiva a partir da etapa de Tecnologia. Diante das circunstâncias de isolamento social, a equipe primeiramente idealizou a realização do mapeamento de tecnologias por meio de formulários online. As perguntas contidas nesses formulários foram elaboradas pela equipe técnica da Instituição e enviadas aos especialistas externos convidados indicados pela própria equipe.

Entretanto, a adesão a este modelo foi baixa, cerca de 30\%. A equipe facilitadora acredita que a baixa adesão é advinda de 2 motivos principais: o formato das questões adotado no formulário que demandou respostas discursivas e o cenário social vivenciado no momento do envio (transição e necessidade de rápida adaptação do presencial para o remoto). 
Desta forma, a fim de viabilizar a continuidade da construção do roadmap durante o isolamento social, a equipe facilitadora organizou e promoveu encontros telepresenciais por meio de webinars realizados pela plataforma da Microsoft ${ }^{\circledR}$ Teams, onde cada encontro demandou um roteiro específico de trabalho contendo perguntas técnicas relacionadas ao escopo da etapa, com duração aproximada de 2,5 horas. Assim, a transição dos tradicionais workshops para o formato webinars consistiu em um grande desafio, entretanto se mostrou eficiente, respeitando suas limitações.

Os principais aprendizados advindos da utilização de ferramentas digitais foram:

- Necessidade de adaptabilidade adequada visto que as dinâmicas remotas são mais recentes e menos estabelecidas.

- Maior necessidade de formas de engajamento durante os encontros como representações visuais e dinâmicas durante o webinar, visto que no ambiente online as pessoas estão mais propensas a dispersão e a realizarem outras atividades concomitantes.

- Encontros com duração mais curta comparado aos presenciais.

- Possibilidade de convidados de diferentes regiões.

- Economia de budget.

- Eventos online geralmente possuem maior adesão pois não contam com o fator deslocamento e facilitam o ajuste das agendas.

\subsection{Transformação Decorrente do Trabalho em Telepresença}

O espaçamento de 10 anos revelou a utilização de novas abordagens na construção do roadmap para manter-se como uma ferramenta adequada de gestão. Os encontros da etapa de tecnologia do ano de 2010 foram realizados no formato de workshop presencial enquanto o ano de 2020 adotou o formato de webinar. Essa transformação impôs que alterações fossem feitas visando a adaptação ao ambiente telepresencial.

Entre estas alterações, ressalta-se: No ciclo mais antigo o encontro de Tecnologia foi realizado em apenas 1 etapa com duração de até 1 dia de trabalho e até 10 convidados dedicados a espaço de fala concomitante. Já no ciclo mais recente optou-se pela divisão em 2 
etapas, uma em cada dia, com até 2,5 horas de duração e até 5 convidados dedicados a espaço de fala concomitante.

Esta transformação ocorreu devido a diferenças de estímulos entre atividades presenciais e telepresenciais, no qual requer diferentes formas de controle. Em ambientes telepresencias, a ausência física requer mecanismos de gestão mais concentrados no ponto de atenção dos participantes, a fim de evitar dispersão e realização de demais atividades concomitantes sem relação com o tema.

Observa-se que o principal aprendizado relacionado a abordagem de trabalho foi:

- Os encontros telepresenciais são melhor aproveitados se controlado o número de participantes por evento. Nota-se que encontros telepresenciais com até 5 convidados dedicados a espaço de fala, são mais eficientes em tempo e aprofundamento dos temas (este número não contempla os participantes ouvintes). Este número é flexibilizado nos encontros presenciais, que permitem um número maior de participantes sem comprometer o resultado.

\subsection{Variação do Esforço de Investimento}

Frente às transformações vivenciadas, foi possível estimar um comparativo de esforço financeiro relativo à quantidade de profissionais envolvidos em cada etapa marco de cada ciclo e o tempo de dedicação de cada profissional nos encontros (homem-hora, HH), para isso, considerou-se como premissa para o cálculo:

- O universo amostral refere-se aos participantes da Instituição que formam a equipe técnica, profissionais de nível tático e nível estratégico da Instituição. ${ }^{4}$

- A quantificação das horas refere-se à dedicação nos encontros de Mercado, Produto, Tecnologia e reuniões de trabalho adicionais. ${ }^{5}$

Para auxiliar na análise, os profissionais foram divididos em 3 níveis distintos, considerando a diferenciação por faixa salarial.

\footnotetext{
${ }^{4}$ Custos com participação de profissionais externos à Instituição não foram considerados.

${ }^{5}$ As etapas de Planejamento, Pesquisa e Mapeamento não foram contempladas.
} 
- Estratégico: Gerentes e profissionais da alta administração da empresa, com visão dos objetivos gerais.

- Tático: Coordenadores e profissionais com visão por Instituto.

- Operacional: Especialistas e analistas responsáveis por tarefas rotineiras.

A Tabela 4 e 5 a seguir apresentam a quantidade de profissionais envolvidos de acordo com sua faixa de atuação e as horas dedicadas aos projetos em 2010 e 2020 respectivamente. Desta forma, é possível estimar o valor aproximado do esforço de investimento financeiro.

TABELA 4 - Participação HH por faixa profissional em 2010.

\begin{tabular}{cccc}
\hline Faixa profissional & $\begin{array}{c}\text { Quantidade de } \\
\text { profissionais }\end{array}$ & $\begin{array}{c}\text { Horas dedicadas } \\
\text { por profissional }\end{array}$ & Total HH \\
\hline Estratégico & 2 & $32 \sim$ & 64 \\
\hline Tático & 2 & $32 \sim$ & 64 \\
\hline Operacional & 8 & $25 \sim$ & 200 \\
\hline
\end{tabular}

TABELA 5 - Participação HH por faixa profissional em 2020.

\begin{tabular}{cccc}
\hline Faixa profissional & $\begin{array}{c}\text { Quantidade de } \\
\text { profissionais }\end{array}$ & $\begin{array}{c}\text { Horas dedicadas } \\
\text { por profissional }\end{array}$ & Total HH \\
\hline Estratégico & 1 & 3 & 3 \\
\hline Tático & 5 & 25 & 125 \\
\hline Operacional & 7 & 32 & 224 \\
\hline
\end{tabular}

Observa-se neste contexto, que o tempo de contribuição de profissionais de nível estratégico em 2020 foi menor quando comparado com o ano de 2010, sem perder a qualidade do trabalho desenvolvido. Isto foi possível devido a forma de trabalho telepresencial assumida no novo ciclo, que permite ao profissional de nível estratégico participar apenas nos momentos de tomada de decisão, flexibilizando a carga horária de trabalho.

Por outro lado, como a mesma forma de trabalho telepresencial permite a presença em maior número de profissionais, foi observado no aumento de participantes de nível tático e por maior tempo de colaboração, se comparado com o ano de 2010, visto que a distância geográfica e eventuais tempos de deslocamentos são eliminados frente a este recurso.

Além da melhor gestão e consequente redução do valor de investimento com custos diretos (designado investimentos em $\mathrm{HH}$ ), outra influência na variação do investimento está 
relacionada a redução dos custos indiretos (designado gastos com deslocamento, hospedagem e alimentação), no qual foi possível com a realização de eventos online.

Desta forma, observa-se que o principal aprendizado relacionado a variação do esforço de investimento foi:

- Melhor gestão e consequente redução do esforço de investimento relativo a custos diretos no ano de 2020 em relação a 2010, viabilizado pela flexibilização do tempo de participação dos profissionais perante suas atribuições e responsabilidades.

- Gasto evitado com custos indiretos, como transporte e hospedagem de convidados e eventuais buffet de coffe break foi possível por meio dos encontros telepresenciais.

\subsection{Renovação do Modelo de Entrega}

Alinhado ao mundo mais tecnológico e com a necessidade de constantes atualizações do Roadmap, o formato da entrega do mapeamento passou por uma grande transformação. Optou-se por realizar o mapeamento das Rotas Tecnológicas de forma mais interativa e digital, possibilitando ao leitor uma experiência mais participativa.

Desta forma, a concisão deste processo construtivo apresenta-se no formato de Plataforma Interativa. Uma estrutura onde o leitor pode escolher por onde navegar e o que visualizar no Mapa de Rotas: Direcionadores de Mercado, Plataformas Tecnológicas, Direcionadores de Tecnologia ou a visualização global.

A apresentação em uma Plataforma Interativa foi pensada considerando os conceitos de experiência do usuário e garante fácil acesso, fluidez na navegação, interatividade e permite fácil atualização, quando necessário. Ressalta-se que a plataforma foi elaborada com o auxílio do Pacote Adobe ${ }^{\circledR}$ listado anteriormente. A Plataforma Interativa com o resultado do mapeamento do ciclo 2021-2025 pode ser acessada pelo link: http://bit.ly/MapadeRotas_QMA.

Ressalta-se que as Rotas Tecnológicas 2011-2015 foram estruturadas com base no formato "genérico" proposto por EIRMA (1997) e também passaram por adaptações para melhor atender aos objetivos da empresa, no entanto, difundida com maior relevância no ambiente interno da Instituição, visto que, embora formatada em meio digital, a multiplicação do conteúdo foi majoritariamente em formato físico. 
Por fim, os principais aprendizados relacionados ao modelo de entrega foram:

- A diagramação e apresentação exclusivamente em meio digital possibilitou multiplicação do conteúdo além da facilidade na leitura, visto que este pode ser livremente navegável mediante interesse do leitor.

- Durabilidade indeterminada do material desenvolvido.

- Facilidade na acessibilidade aos stakeholders.

- Maior facilidade para atualização dos dados para continuidade do roadmap dos próximos ciclos.

\section{CONCLUSÃO}

Este estudo comparou o resultado do Technology Roadmapping, com aplicação presencial realizado em 2010 com a aplicação telepresencial realizada em 2020, e indicou aprendizados resultantes destas aplicações relacionados a possibilidade de atuação com uma equipe multidisciplinar e seus ganhos em análise crítica, consequente identificação de novas ferramentas de trabalho direcionadas ao uso digital, percepção de desejável limitação de profissionais com colaboração concomitante em ambiente de telepresença para garantir a qualidade do conteúdo trabalhado, melhor flexibilização de acesso mediante telepresença que permite melhor gestão dos recursos humanos e redução de custos indiretos e por fim, expansão do conteúdo mapeado e liberdade de acesso ao roadmap.

A metodologia empregada tanto presencial quanto telepresencial mostrou-se satisfatória diante do momento vivido em ambos os ciclos, cumprindo os objetivos propostos em cada situação. Desta forma, conclui-se que as duas propostas possuem espaço para aplicação, resguardado as necessidades de adaptação dos processos para melhor aproveitamento em cada abordagem, onde um modelo híbrido, considerando o contexto de utilização, o ambiente e os objetivos de cada empresa pode servir como o mais adequado relacionado as mudanças e tendências mundiais.

\section{AGRADECIMENTOS}

Os autores agradecem o suporte técnico dos profissionais do Instituto SENAI de Tecnologia Química e Meio Ambiente e Instituto SENAI de Inovação em Química Verde da Firjan, pelo 
período de intensa dedicação e constantes transformações diante dos reflexos causados pela pandemia do covid-19 e pela generosa participação dos especialistas externos convidados que contribuíram para enriquecimento da pesquisa.

\section{REFERÊNCIAS}

BENYON, D.; RESMINI, A. User Experience in Cross-channel Ecosystems. In: $31^{\text {st }}$ British Human Computer Interaction Conference, 2017, Sunderland, Anais.

EIRMA (European Industrial Research Management Association). Technology Roadmapping - delivering business vision. Relatório Técnico. Paris, 1997.

GARCIA, M.L.; BRAY, O.H. Fundamentals of technology roadmapping: Relatório Técnico. Estados Unidos, 1997.

GIORDANO, C.S.S. Technology Roadmapping (TRM) como método de planejamento e gestão: O caso do centro de tecnologia SENAI Ambiental. 2011. 170f. Dissertação (Mestrado em Tecnologia de Processos Químicos e Bioquímicos) - Programa de Pós Graduação em Tecnologia de Processos Químicos e Bioquímicos, Universidade Federal do Rio de Janeiro, Rio de Janeiro, 2011.

KERR, C., PHAAL, R. Technology roadmapping: Industrial roots, forgotten history and unknown origins. Technological Forecasting \& Social Change v. 155, 2020.

PHAAL, R., FARRUKH, C., PROBERT, D. Technology roadmapping: linking technology resources to business objectives. University of Cambridge, p. 1-18, 2001.

PHAAL, R.; FARRUKH, C.; PROBERT, D. Developing a technology roadmapping system. In: Portland International Conference on Management of Engineering and Technology, n5, 2005, Portland, Anais, p.99-111. 
RENZI, A. B. et al. User experience journey with multiple devices and telepresence during quarantine. Ergodesign \&amp; HCI, [S.1.], v. 8, n. 1, p. 80-95, june 2020.

SCHLEMMER, E. Telepresença. Curutiba: IESDE Brasil S.A, 2009.

UNIDO - UNITED NATIONS INDUSTRIAL DEVELOPMENT ORGANIZATION. UNIDO technology foresight manual. v. 1, Vienna, 2005.

WEFORUM - World Economic Forum in collaboration with Deloitte. Technology Futures: Projecting the Possible, Navigating What's Next: relatório, 2021. 